\title{
IUFOST2006/1352 High Pressure Assisted Structure Engineering of Foods
}

\author{
M. Hendrickx, A. De Roeck, T. Duvetter, I. Fraeye, D. Sila, C. Smout, S. Van Buggenhout, A. Van Loey \\ and I. Verlent \\ Laboratory of Food Technology, Kasteelpark Arenberg 22, B-3001 Heverlee, Belgium \\ marc.hendrickx@biw.kuleuven.be
}

\begin{abstract}
For more than one decade, food processing and preservation technologies based on the use of high hydrostatic pressure are a focus point of research and development in the field of food science and technology. During the same period, a number of industrial applications have been implemented. For plant based foods, relations between (bio) chemically induced molecular changes of pectin, tissue adhesion and texture changes during processing have been indicated, in particular for thermal processing. This contribution re-evaluates the existing know how and integrates recent findings in the context of high pressure processing (including high pressure as a pre-treatment technology, high pressure thermal processing and high pressure low temperature treatments). Effects on key enzymes (activity and inactivation) such as pectin methylesterase and polygalaturonase and associated pectin changes as well as chemical pectin changes during processing are discussed in detail and related to texture changes of high pressure treated plant based food products.
\end{abstract}

